\title{
Job satisfaction among British physician associates
}

\author{
Authors: Tamara S Ritsema ${ }^{A}$ and Karen A Roberts ${ }^{B}$
}

\begin{abstract}
All British physician associates (PAs) were invited to participate in the annual census of the UK Association of Physician Associates (UKAPA) in May 2014. Each participant completed the Cooper 10-item Job Satisfaction Scale and a PA-specific job satisfaction survey. In general, PAs were found to be satisfied with their work. No factor assessed by the survey had lower than a $66.6 \%$ satisfaction rate. Of the factors assessed, PAs were most satisfied with their relationships with the doctors on their teams. They were least satisfied with their ability to use their training and abilities, with only $66.6 \%$ of participants reporting satisfaction with this aspect of their work. Like their American colleagues, British PAs are generally satisfied with their work. They are least satisfied with their ability to fully use their training, which is likely due to the youth of the profession, lack of prescriptive rights and lack of understanding of the PA role.
\end{abstract}

KEYWORDS: Job satisfaction, physician assistant, physician associate, role satisfaction

\section{Introduction}

Physician associates (PAs) are health professionals trained to obtain a health history, perform comprehensive physical examinations, interpret laboratory and radiologic investigations, perform diagnostic and therapeutic procedures, render diagnoses and initiate treatment. ${ }^{1}$ PAs are trained in the generalist medical model and practise in both primary and secondary care settings. While the intensity of doctor supervision can vary based on the setting and the experience of the PA, all PAs always practise under the supervision of a fully-qualified doctor. PAs in the USA (where they are called 'physician assistants') have been practising medicine with doctor supervision since 1967. Currently, approximately 100,000 American PAs practise in all medical and surgical

Authors: A assistant professor, George Washington University School of Medicine and Health Sciences, Washington DC, USA and honorary senior lecturer, Physician Associate Programme, St George's, University of London, London, UK; Breader and course director, Physician Associate Programme, St George's, University of London, London, UK specialties. ${ }^{2}$ In the USA, PAs can prescribe medications and can also order radiologic investigations.

In the early and mid-2000s, pilot projects using US-trained PAs in the West Midlands and Scotland were initiated. Evaluations of these pilot projects were generally positive and led several universities to establish PA training courses in the late 2000s. In addition, the Department of Health developed and published the Competence and curriculum framework for the physician assistant outlining the competencies in which British PAs should be educated. ${ }^{1}$ By the end of 2014, five UK universities were training PAs and up to 20 more were planning new courses. ${ }^{3}$ In total, 191 PAs were practising in a wide variety of medical and surgical specialties across the UK. ${ }^{4}$ Because the PA profession in the UK does not yet have statutory regulation, PAs are not currently eligible to prescribe or request investigations that use ionising radiation, and the title is not protected from those who might wish to misrepresent themselves as PAs.

Research on PAs in the USA has demonstrated that PAs are very satisfied with their roles and their jobs. ${ }^{5-7}$ In all surveys, PAs are more satisfied with their work than doctors are. ${ }^{8,9}$ However, the degree to which British PAs are satisfied with their role and their employment has never been investigated. It is possible that US PAs are satisfied with their role in part because PA practice in the USA has few limits, whereas PA practice in the UK is limited by the lack of experience of doctors and the health system with the role, lack of statutory regulation, and lack of defined scope of practice. To try to answer these questions, we developed a survey on PA job and role satisfaction.

\section{Methods}

The UK Association of PAs (UKAPA), now the Faculty of Physician Associates (FPA) at the Royal College of Physicians, has commissioned an annual census of all PAs and PA students in the UK since 2011. This survey collects demographic, specialty and salary data, and information on scope of practice for each PA. Each PA can be tracked longitudinally, but in a de-identified fashion, through the use of a census unique ID. All PAs living in the UK were invited to participate in the fourth annual UKAPA census in May 2014. ${ }^{4}$ This study received ethical approval from the Institutional Review Board at the University of Nebraska Medical Center. 
The Cooper 10-item Job Satisfaction Scale (JSS) was administered to all practising PAs who completed the 2014 UKAPA Census. This survey, which assesses 10 domains (Table 2) on which health professionals may or may not be satisfied with their work, was first developed in 1989 and was validated in $2012 .{ }^{10,11}$ No changes were required to make the questions applicable to PAs and the survey was administered in the same way Hills et al administered the survey to Australian general practice physicians in the 2012 validation study. ${ }^{11}$

In addition, another job satisfaction survey was developed de novo using the literature as a guide to satisfaction factors specific to PAs. For each of the 10 PA-specific dimensions of job and role satisfaction, participants were asked both a positively and negatively-worded question to assure that participants were reading the questions carefully and answering consistently. For example, one pair of questions were 'On the whole, the doctors with whom I work treat me with respect' and 'I often feel disrespected by the doctors with whom I work'. Participants were asked to choose 'strongly disagree', 'disagree', 'agree' or 'strongly agree' for each statement presented.

As PAs practise within a unique relationship to doctors, four of the 10 dimensions assessed were about the PAs' relationships with their supervising doctors. Other dimensions included degree of autonomy in practice, opportunities for ongoing training, time spent with patients, whether the PA was satisfied with his/her clinical specialty, quality of care for patients and ability of PAs to be used to their full potential (Table 3). These items were pre-tested with groups of both US- and UKtrained PAs for general understanding and face validity, but the instrument itself has not been validated. The responses to positively and negatively worded questions for each domain were analysed for consistency of response. Dimensions in which the positively-worded and negatively-worded questions had appropriate opposing responses at least $96 \%$ of the time were included. Those dimensions which agreed less than $96 \%$ of the time were not included in the analysis. Results were analysed using IBM SPSS Statistics version 22.0.

\section{Results}

All PAs living in the UK were invited to participate in the fourth annual UKAPA census in May 2014. Of the 191 PAs living and working at this time in the UK, 135 completed the survey $(70.6 \%)$. Of the 135 respondents to the census, $124(92 \%)$ completed the entire job satisfaction portion of the survey. Respondents practised in 30 different medical and surgical specialties all across England and Scotland. The median number of years of experience as a PA was 3 (range: 4 months to 29 years). $71 \%$ of the respondents were women and $29 \%$ were male (Table 1 ).

The Cooper 10-item JSS was administered to each participant. Of the 135 respondents, 124 completed the scale. Each satisfaction item is scored from 0 (very dissatisfied) to 4 (very satisfied). The range of scores was 3-40, with a mean of 29.6 and a median of 30 (Table 2). Nine respondents answered 'very satisfied' for all questions and thus had a JSS score of 40; 12 participants had total JSS scores of less than 20 . The correlation $\left(r^{2}\right)$ between the overall scale score and the score on the question 'Taking everything into consideration, how do you feel about your work?' was 0.85 . Respondents were most satisfied with their colleagues (mean 3.37) and hours of work
Table 1. Characteristics of physician associates responding to the 2014 UKAPA Census

\begin{tabular}{ll} 
Characteristics & $\begin{array}{l}\text { Number of } \\
\text { respondents (\%) }\end{array}$ \\
Female & $96(71)$ \\
Male & $39(29)$ \\
Specialty & \\
General practice & $31(23)$ \\
Emergency medicine & $22(16)$ \\
Acute medicine & $20(15)$ \\
Trauma and orthopaedics & $11(8)$ \\
Other (mostly hospital-based specialties) & $51(38)$ \\
Length of time as a PA & \\
$0-1$ year & $28(21)$ \\
2 years & $32(24)$ \\
3 years & $23(17)$ \\
4 years & $15(11)$ \\
5 years & $16(12)$ \\
$>5$ years & $21(16)$ \\
(Range 0-29 years, median 3 years) & \\
\hline UKAPA = UK Association of Physician Associates; PA = physician associate
\end{tabular}

(mean 3.04), and least satisfied with the opportunities they had to use their abilities (mean 2.75) and their pay (2.62). The mean overall level of satisfaction was 3.03 , which correlates with the description 'moderately satisfied'.

The newly developed PA-specific job satisfaction scale was administered to the same participants as those who completed the JSS. Responses were counted as positive when the respondents chose 'agree' or 'strongly agree'. Respondents

Table 2. Physician associate job satisfaction according to the Cooper 10-item Job Satisfaction Scale

\section{Domain}

Colleagues

Hours of work

Overall satisfaction

Physical working conditions

Freedom to choose my own way of working

Amount of responsibility

Variety of work

Recognition for good work

Opportunity to use abilities

Pay

Mean overall score 29.6, median 30, range 3-40 
to these questions were generally found to have a good relationship with their supervising doctors; they felt respected (98\%), supported when they needed the doctor's help (94\%), and appreciated for their contributions to the team (93\%). PAs were less satisfied with the level of autonomy they have in their role as a PA ( $85 \%$ felt the level was appropriate) and doctor understanding of the PA role (84\%). Only $66 \%$ of PAs felt that they were used to their full potential on the team.

\section{Discussion}

In general, British PAs are reasonably satisfied with their work. The results of the JSS showed that mean and median overall score equaled 'moderately satisfied' on the JSS Likert scale. The mean score of 29.6 is slightly lower than the mean JSS score in the Australian GP population (mean 30.4). ${ }^{11}$ PAs report relatively lower satisfaction with pay and opportunities to use their skills and relatively higher satisfaction with colleagues according to the JSS. The satisfaction rates found on the PA-specific job satisfaction scale were similar. Even on the lowest scored domain (used to full potential), 66\% of PAs were satisfied or very satisfied. The results between the two scales were concordant. All three of the highest scoring domains on the PA-specific scale related to PA satisfaction with their relationships with the doctors with whom they work. These results are concordant with the JSS in which 'satisfaction with colleagues' ranked highest. In both cases, satisfaction with the ability of the PA to be used to their full potential was among the lowest domains.

Neither the JSS nor the PA-specific scale can delineate all of the possible reasons PAs are satisfied or unsatisfied in certain domains. However, it is clear that a substantial minority of PAs do not feel they are being used as effectively as possible within the clinical team. Some possible reasons for this include that as unregulated medical professionals, they are not eligible for prescribing privileges and are not permitted to request investigations that use ionising radiation. As a new profession, PAs often have to explain themselves and their training repeatedly to members of the healthcare team. No one in the NHS has 20 or 30 years of experience with PAs to rely on to educate doctors and other healthcare providers about the potential uses and growth of the role over time, which may lead to under-use of the PAs' training. Over time, it is possible that with the growth, maturation and legal recognition of the profession, PAs will begin to be allowed broader practice more in keeping with their training.

\section{Limitations}

This study has several limitations. First, the number of PAs in the UK is very small, which limits the possibilities for more detailed statistical or subgroup analyses. We were not, for example, able to compare satisfaction by specialty, pay, practice setting or number of years' experience as a PA. Second, the survey assesses levels of satisfaction with specific domains but does not assess all of the possible reasons for job or role satisfaction. To better understand all of the possible reasons for job and role satisfaction, we would need to undertake a detailed qualitative analysis. Third, while the 10-item JSS has been validated in the GP population, it has not been validated with PAs. However, the fact that the PA responses were very similar to the GP responses in the article by Hills et a ${ }^{11}$ does lend some credibility to the idea that this may be a useful instrument for assessing PA satisfaction because, like GPs, PAs are trained as generalists. Finally, the PA-specific questionnaire developed for this study was pre-tested and modified based on the pre-testing, but the instrument has not been validated. Three of the ten domains failed the test of concordance between the positively and negatively-worded questions. The wording of these questions likely needs to be more precise to make sure the nuance of each captures the same aspects of the domain in the minds of the respondents.

\section{Conclusion}

Like American PAs and Australian GPs, British PAs are generally satisfied with their jobs. They are very satisfied with the relationships they have with doctors and are less satisfied with the degree to which they are able to use their full range of skills. Further qualitative research is needed to delineate all of the sources of satisfaction and dissatisfaction, both with specific jobs and with the role overall. Replicating this study after PAs obtain statutory regulation may shed light on the degree to which legal restrictions impact satisfaction with the role.

\section{Conflicts of interest}

The authors have no conflicts of interest to declare.

\section{References}

1 UK Department of Health. the competence and curriculum framework for the physician assistant. London: DoH, 2006.

2 American Academy of Physician Assistants. History of the PA Profession. Available online at www.aapa.org/threeColumnLanding. aspx?id=429 [Accessed 1 July 2016].

3 Faculty of Physician Associates. FAQs. Available online at www. fparcp.co.uk/faqs/ [Accessed 1 July 2016].

4 Ritsema T. 2014 UKAPA census results. London: UK Association of Physician Associates, 2014.

5 LaBarbera DM. Gender differences in the vocational satisfaction of physician assistants. JAAPA 2010;23:33-4, 36-9.

6 Biscardi CA, Mitchell J, Simpkins S, Pinto Zipp G. Practice characteristics and lifestyle choices of men and women physician assistants and the relationship to career satisfaction. J Allied Health 2013;42:157-62.

7 Labarbera DM. Physician assistant vocational satisfaction. JAAPA 2004;17:34-6, 38-40, 51.

8 Hooker RS, Kuilman L, Everett CM. Physician assistant job satisfaction: a narrative review of empirical research. J Physician Assist Education 2015;26:176-86.

9 The Physicians Foundation. 2014 survey of America's physicians: practice patterns and perspectives. The Physicians Foundation, 2014.

10 Cooper CL, Rout U, Faragher B. Mental health, job satisfaction, and job stress among general practitioners. BMJ 1989;298:366-70.

11 Hills D, Joyce C, Humphreys J. Validation of a job satisfaction scale in the Australian clinical medical workforce. Eval Health Prof 2012;35:47-76.

Address for correspondence: Professor T S Ritsema, Department of Physician Assistant Studies, George Washington University School of Medicine and Health Sciences, 2100 Pennsylvania Ave NW, Suite 369, Washington DC 20037, USA.

Email: tritsema@gwu.edu 